\title{
Hand Vein Infrared Image Segmentation for Biometric Recognition
}

\author{
Ignacio Irving Morales-Montiel ${ }^{1}$, J. Arturo Olvera-López ${ }^{1}$, Manuel Martín-Ortíz ${ }^{1}$, and \\ Eber E. Orozco-Guillén ${ }^{2}$ \\ ${ }^{1}$ Facultad de Ciencias de la Computación \\ Benemérita Universidad Autónoma de Puebla \\ Av. San Claudio y 14 sur. Ciudad Universitaria. \\ Puebla, Pue., Mexico \\ cpycon@hotmail.com, \{aolvera, mmartin\}@cs.buap.mx \\ ${ }^{2}$ Programa de Ingeniería en Informática \\ Universidad Politécnica de Sinaloa \\ Carretera Municipal Libre Mazatlán Higueras Km. 3. \\ Mazatlán, Sin., Mexico \\ eorozco@upsin. edu. mx

\begin{abstract}
In this paper we propose a technique for both finding vein regions from thermal dorsal hand images and extracting features for biometric recognition; our technique analyzes the geometry of the hand to isolate the vein regions and extract some features (vein bifurcations and ending points) for being used as features in the training sets for classifiers. Commonly, the features extracted are used as geometric and descriptive representation of the vein patterns which the person's identity.
\end{abstract} \\ are matched with hand vein images in a database in order to determine/verify
}

Keywords: Biometry, Hand vein recognition, Infrared image segmentation, Dorsal hand vein image processing

\section{Introduction}

It is very common that for security applications, the users must claim their identify in order for accessing to several services. Typical methods for identification use ID cards, passwords, Personal Identification Numbers (PIN), etc. but they are unreliable since they are easy to be either stolen or forgotten. Currently, it is possible to be aimed by technology through Biometric Recognition systems for overcoming these drawbacks.

In Computer Science, Biometric Recognition is the science of identifying a person based on the physiological and/or behavioral characteristics; these characteristics are known as biometrics and for every person, they have special features such as: Universality, Uniqueness, Permanence and Measurability [1]. Some examples (Fig. 1) of typical biometrics are: Iris, face, palm print, signature, finger print, ear structure, among others $[2,3,4]$. 
Biometric Recognition is commonly an automatic technique used in our digital age; in this context, a computer system designed for either person identification or person verification is named biometric system. In person verification, the user claims an identity and the recognition system verifies whether the claim is genuine or not; in person identification the recognition system identifies the user without claiming an identity[1].

A typical biometric systems consist of the following phases:

-Capture. This stage is related to the acquirement of the biometric through a digital capture device or sensor (e.g. camera, scanner, microphone).

-Pre-processing. This module respects to the steps for preparing the information to be analyzed from the biometric. When the biometric is stored via a digital image, typical pre-processing steps are: cleaning (noise), contrast enhancement, sharpen, equalization, among other processes. Pre-processing is an important phase since the better quality of information provided to the biometric system the higher recognition rate is achieved.

-Feature extraction. This stage extracts the most relevant descriptive components (minutiae) that describe to the biometric. The main processes involved in feature extraction are the Region Of Interest (ROI) extraction and segmentation; the goal of both processes is the isolation of the region where there exists relevant and descriptive information about the biometric.

-Recognition. This is the last phase in a Biometric Recognition system and uses the features extracted to either person identification or person verification. Commonly, classifiers (supervised, non supervised) are used in this recognition stage; the main goal of the classifier is to extract (through its learning steps) models or patterns from each biometric in the dataset which are used for recognition purposes.
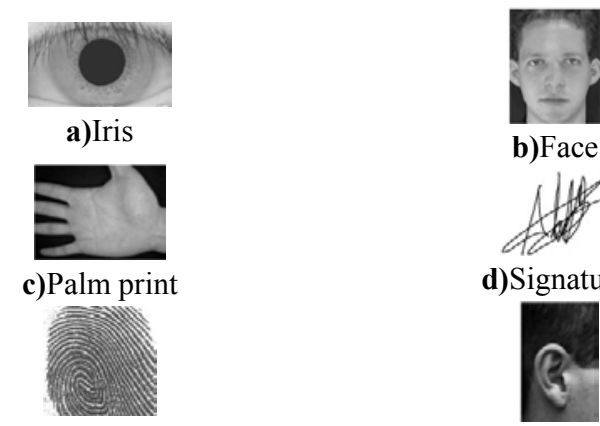

e)Finger print

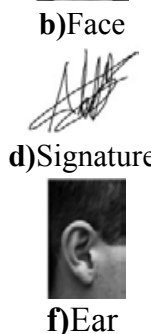

Fig. 1. Biometrics commonly used for person recognition a)Iris, b)Face, c)Palm print, d)Signature, e)Finger print, f)Ear

In this work, we focused in the pre-processing stage, ROI extraction and feature extraction for a Biometric Recognition system based on dorsal hand vein as biometric.

Compared to typical biometrics, vein recognition is a new member of the biometric family. This technology started in the 1990s, but it did not attract much attention in that decade. Since 2000 year, few papers on this topic have been published and nowadays it is an active research field. 
Hand vein images are commonly captured by digital cameras operating in the visible range $(400 \mathrm{~nm}-700 \mathrm{~nm}$ wavelength) of the electromagnetic spectrum i.e. illuminating the region to capture using visible light. In the captured images it is difficult to discern the veins since in some cases the person's veins are not superficially visible.

Fortunately, the Infrared (IR) light $(2.5-50 \mu \mathrm{m}$ wavelength) could help us when veins are not easily visible. IR light is a non visible light that penetrates into the skin about $3 \mathrm{~mm}$. Due to the hemoglobin properties in the veins, it absorbs the infrared radiation and as consequence, the veins are contrasted in a dark color. When IR light is used to capture dorsal vein images, it is necessary to capture via IR cameras which are named thermal cameras and the captured images are named thermal images [5].

One of the main problems using thermal image for recognition is the low contrast in the images, because it is not easy to distinguish components that could be simple to recognize for the human eye. Unfortunately there is not a global solution to this problem, but there are useful methods for specific cases.

It is important to have a special mention about the vein pattern, which is a vast network of blood vessels underneath a person's skin. Like fingerprints, the shape of vascular patterns is believed to be distinct among different people [6-8], and very stable/invariant over a long period of time in most of the cases. In addition, as the blood vessels are hidden underneath the skin and they are mostly invisible to the human eye, vein patterns are more difficult to forge by intruders (in comparison to other biometrics such as signature).

A key part of hand dorsal vein biometric is the difference with other similar biometrics because its recognition requires analyzing alive people, putting special attention that when person is not alive the vein pattern color would change, also the pressure, temperature and other similar characteristics. Hand veins can prevent a possible misuse of users' identity, considering that there are several myths about the misuse with the fingerprints when they do not require vitality by the biometric( e.g. mutilations by intruders that would damage both the users' integrity and users' body).

The properties of hand vein make it a potentially good biometric which offers secure and reliable features for person's identification or verification.

\section{$2 \quad$ Hand vein IR image pre-processing}

As it was mentioned before, it is not easy to obtain a pattern from a raw hand vein thermal image. For this reason, the ROI extraction, segmentation and contrast enhancement are necessary for the recognition step in a Biometric Recognition system. In the following sections, we present an approach for ROI extraction, vein segmentation and feature extraction from hand vein thermal images.

\subsection{ROI extraction}

In Fig. 2, a couple of hand vein thermal images with low contrast are shown. In order to extract the ROI in these low-contrast cases, it is necessary to isolate the hand in the image, so we need to use a mask in order to delimit the hand. 
As initial step, we propose to use a 3x3 Median filter to eliminate the possible noise of the image. After that, to get the position of the hand, we use a Sobel Filter to find the area with the most of edges. After edges are obtained, we apply a horizontal white filling $(6 \mathrm{px})$ that is a simple process which take every white pixel and look at the 6 neighbor pixels left (in this case), and if it finds a white pixel then it fills the pixels to white until that pixel; then a vertical white filling process is applied, which takes every white pixel and fills in white every pixel above (Fig. 3b).

For each column, the mean is calculated to create a histogram and find the valley points (see Fig. 4a) where:

$P 1$ is originally the valley point between the small finger and the ring finger.

$P 2$ is originally the valley point between the index finger and the thumb.
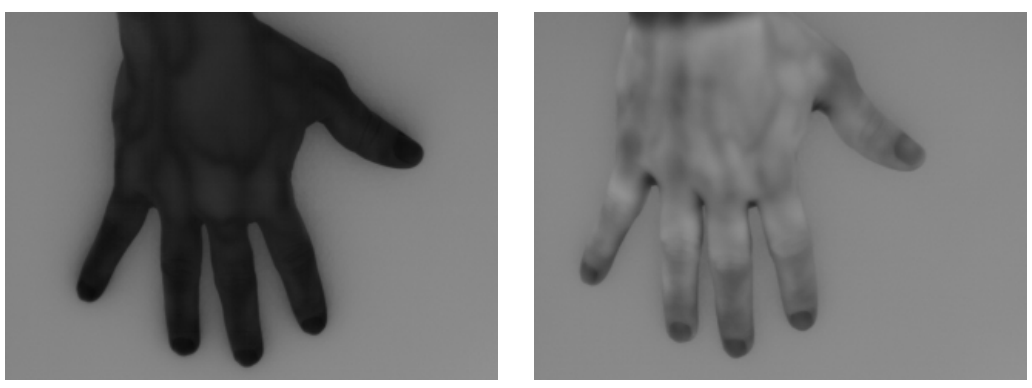

Fig. 2. IR hand vein images of two hands captured in different environments

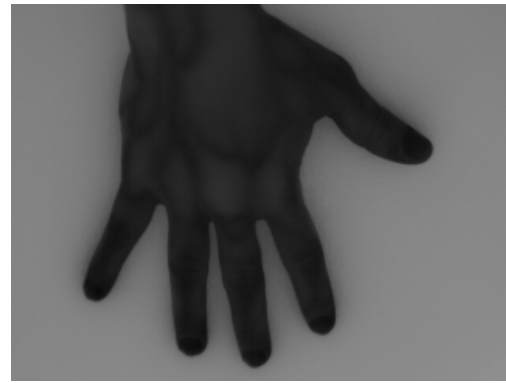

a)

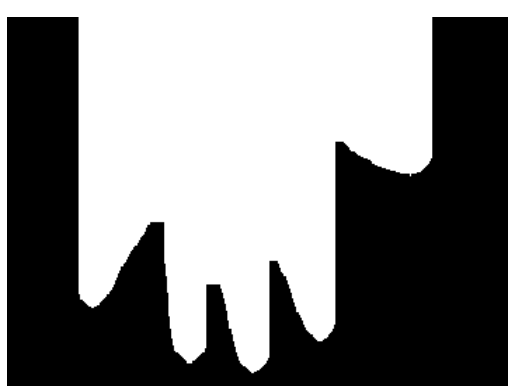

b)

Fig. 3. Creating the Mask. a)IR hand vein image. b) Hand Mask obtained from a)

The Euclidean Distance $D$ between $P 1$ and $P 2$ is calculated to be used as a reference of the hand dimensions. Then point $P 2$ is adjusted to estimate the position of the valley point between the middle finger and the index finger ( $X$ position is $D * 4 / 7$ from $P 1$ and $Y$ position is at the same height than $P 1-D / 7) . P 1$ is adjusted to estimate the actual position of the valley point between the middle finger and the index finger $(Y$ position is $Y+D / 1.4$ ). 
The ROI is therefore defined as a rectangular region $R_{P 1 P 2 P 3 P 4}$ (Fig. $4 \mathrm{~b}$ ). Where $\overline{P 1 P 3}=1.4 * \overline{P 1 P 2}$. The process of defining the ROI is shown in Fig. 3 and Fig. 4.

The reason for extracting the ROI in this way is because it ensures all the ROIs reference to the same region in the hand image, and it is irrelevant to the size of the hands; in addition, extracting ROI based on hand landmark will increase the tolerance of the system against hand rotation and low contrast.

The image depicted in Fig. $4 \mathrm{~b}$ shows the result of the extracted ROI from the hand image in Fig. 3a.

\subsection{Image enhancement and segmentation}

The vein patterns show different clearness among different images. That is why it is necessary to enhance the contrast in the images, for this purpose, we propose to apply a $5 \times 5$ Median Filter to remove the speckling noise in the images. Then a 2-D Wiener filter as suggested in [9] was applied to the ROI in order to eliminate the high frequency noise. A Wiener filter is useful as lowpass-filter in the grayscale image in order to eliminate noise. Wiener filtering is based on using pixel neighborhoods of size $m \times n$ to estimate the local mean $(\mu \mathrm{l})$ and local standard deviation $(\sigma \mathrm{l})$. After computing $\mu_{1}$ and $\sigma_{1}$, a filtered pixel $b(i, j)$ is created according to (1) where $v^{2}$ is the average of all the local estimated variances. In this paper a $2 \mathrm{D}$-wiener filter is used in $4 \times 4$ neighborhood regions over the image $I(x, y)$; the noise is assumed to be additive noise (Gaussian white noise).

$$
b(i, j)=\mu_{l}+\frac{\sigma_{l}^{2}-v^{2}}{\sigma_{l}^{2}}\left(I(i, j)-\mu_{l}\right)
$$

Due luminosity changes in the hand vein images, a global thresholding is not useful for separating the vein pattern from the background i.e. vein segmentation. In order to segment dorsal hand vein images it is necessary to apply an adaptive thresholding algorithm as suggested in $[9,10]$, which dynamically uses different threshold values for every pixel in the image based on the analysis of its surrounding neighbors.

For every pixel in the image, its threshold value is set as the regional mean value subtracted by a global offset. To determine the local threshold the expressions (2), (3) and (4) are used; where $m(x, y)$ is the local mean, $\partial(x, y)$ is the local deviation, $w>0$ is the size of the area around the pixel and $\mathrm{k} \in[0,1]$ is a bias which is a control for the level of adaptation varying threshold value.

$$
\begin{gathered}
m(x, y)=\frac{1}{w^{2}}\left(\sum_{i=x-w / 2}^{x+w / 2} \sum_{j=y-w / 2}^{y+w / 2} I(i, j)\right) \\
\partial(x, y)=I(x, y)-m(x, y) \\
T(x, y)=m(x, y)\left[1+k\left(\frac{\partial(x, y)}{1-\partial(x, y)}-1\right)\right]
\end{gathered}
$$

For segmenting the hand image, $T(x, y)$ is used as threshold binarization using $w=11$. 
In Fig. 5b the vein region image after ROI enhancement with noise reduction and normalization is shown. Fig. 5c shows the enhanced ROI after applying the local thresholding binarization.

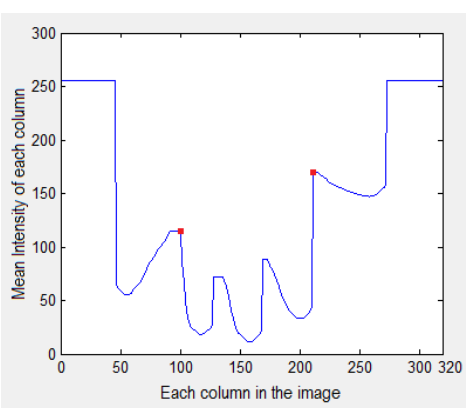

a)

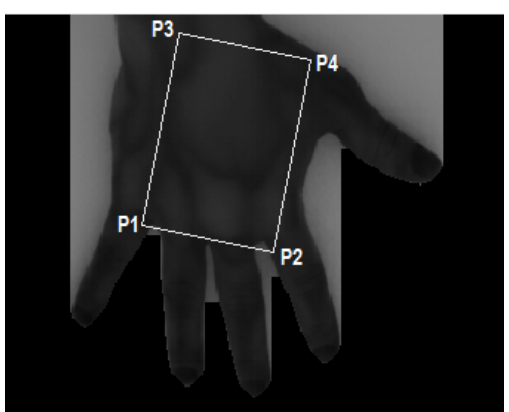

b)

Fig. 4. a)Intensity Histogram. b) Locating the ROI.

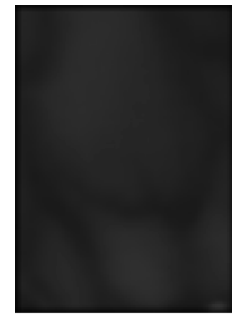

a)

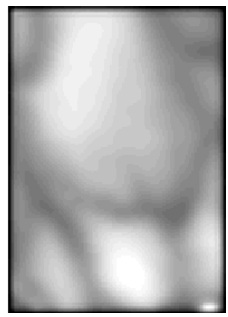

b)

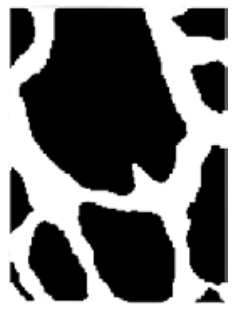

c)

Fig. 5. Preprocessing of IR vein pattern images. a)ROI for the hand image in Fig. 3a). b) After noise reduction and normalization. c) After local thresholding binarization

Table 1. Features of the ETIP- 7320 P-Series Infrared Camera Highlight

\begin{tabular}{|l|l|}
\hline Detector & Microbolometer $320 \times 240$ UFPA VOX \\
\hline Spectral Response & 7 to 14 microns \\
\hline Video Update Rate & $50-60 \mathrm{~Hz}(16 \mathrm{bit}$ digital) \\
\hline Accuracy & $\pm 1^{\circ} \mathrm{C}$ or $\pm 1 \%$ \\
\hline Thermal Sensitivity & $27 \mathrm{mk}$ \\
\hline Operating Temperature & $-20 \mathrm{C}$ to $50 \mathrm{C}$ \\
\hline
\end{tabular}




\subsection{Feature Extraction}

Once the hand vein images have been segmented it is necessary to extract the main descriptive features (minutiae) for each vein pattern from the image since the features extracted are the baseline in the training sets for the classifiers in the recognition step. As result of the segmentation process, the hand vein images are binary but they can be thinned in order to reduce each vein region to a minimal structure and as result the feature extraction process is applied over vein regions of at most one pixel width. For thinning we used the algorithm based on connectivity skeleton proposed in [11].

The main minutiae points that describe the shape and structure of a hand vein are the bifurcation points (i.e. intersection or cross points between veins) since their amount and position are unique for each person; these bifurcation points are quite similar to delta points in fingerprint images. In this work we propose extracting both bifurcation (as proposed in [12] but applied to hand dorsal veins) and end points of the vein.

According to the characteristics of a bifurcation point, a set of masks is used to find a bifurcation over a vein region; the masks used in this work are shown in Fig. 6, these masks uses as pivot the pixel in the center and matching them, either bifurcation or end points over a vein region are found.
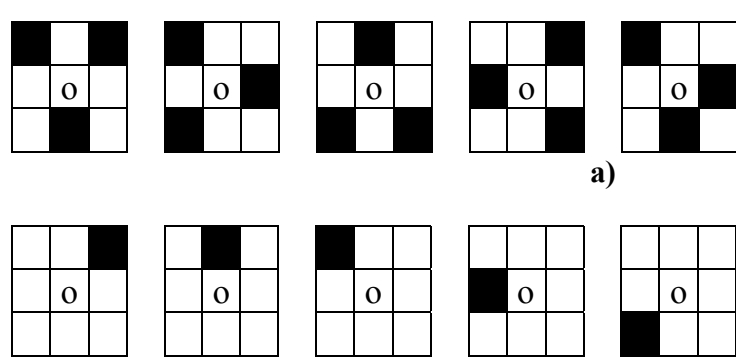

b)

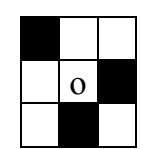

a)
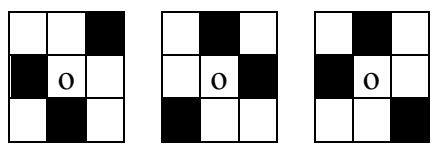

Fig. 6. Masks used for: a)Finding bifurcation points. b)Finding end points. In both cases the pivot is the center pixel in the $3 \times 3$ region

\section{Experimental Results}

For applying our ROI extraction and segmentation approach, we used IR hand vein images with different levels of contrast. These images were captured via an ETIP 7320 P-Series Infrared Camera described in Table 1. 

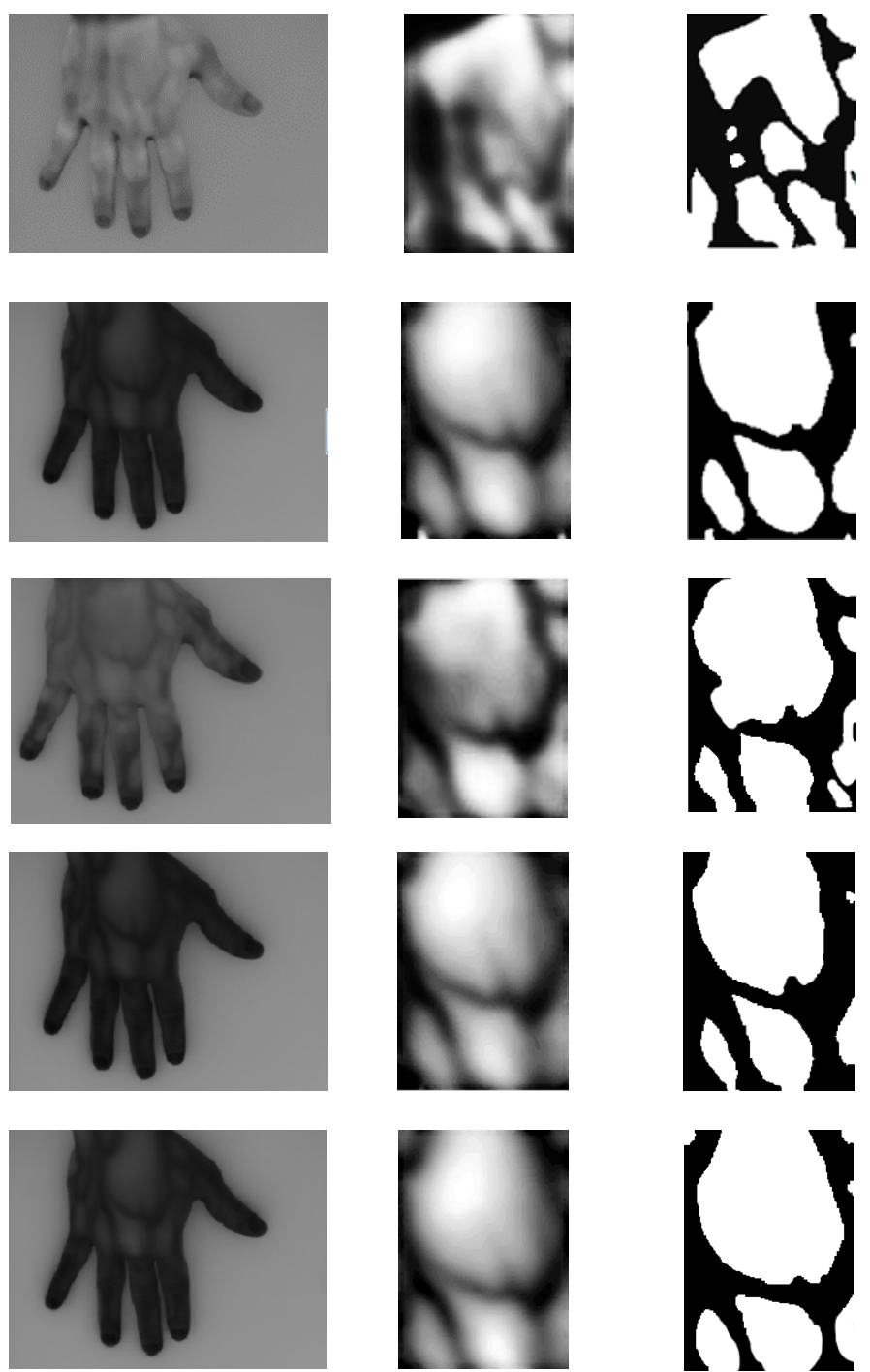

a)

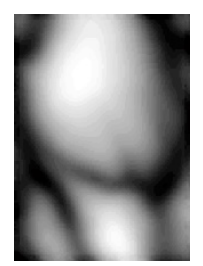

b)
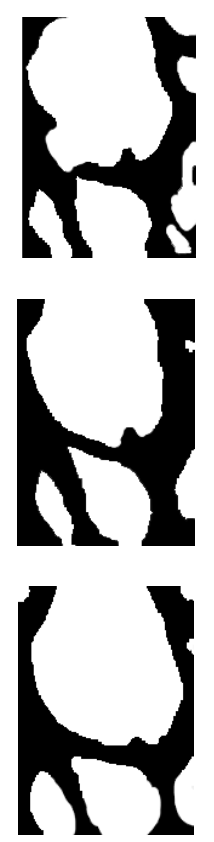

c)

Fig. 7. Results obtained for segmenting IR hand vein images. a) Original IR hand vein image. b) After ROI extraction, noise reduction an normalization. c) After local thresholding

Some results obtained by our ROI extraction and segmentation approach are shown in Fig. 7, where it can be noticed that our approach is able to segment and isolate the ROI related to dorsal hand vein IR images with different low contrast levels which is the main problem to face when trying to analyze biometric hand vein digital images.

A second stage in our experimentation consists in applying our whole approach i.e. ROI extraction, segmentation and feature extraction, as was explained in section 2. 
For these experiments we used the Tecnocampus thermal hand dataset [13]. In Fig. $8 \mathrm{a}$, some of the dorsal hand thermal images (false thermal color) from this dataset are depicted; for our experiments the images were firstly filtered applying negative and gray scale conversion respectively (Fig. 8b).
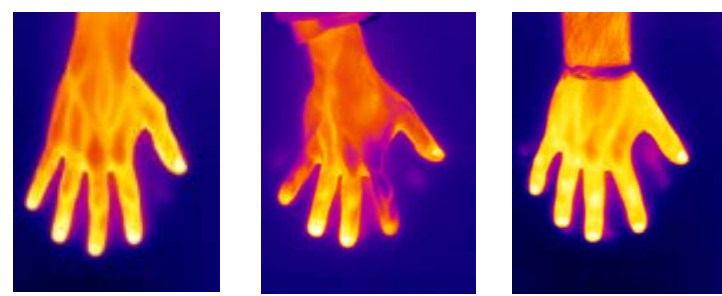

a)
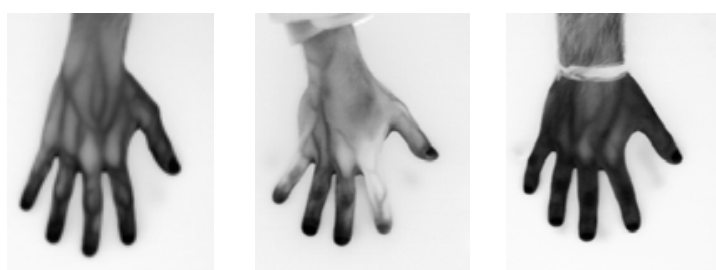

b)
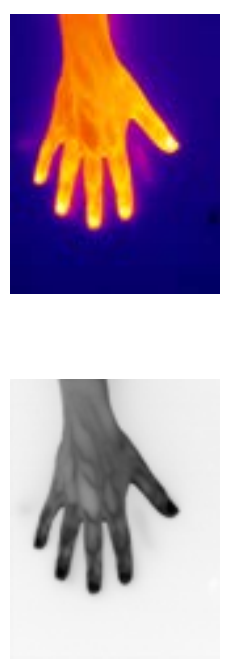

Fig. 8. a)Thermal hand datasets obtained from[13]. b) Filtered images applying negative and gray scale conversion

Some of the results obtained by applying our whole approach are shown in Fig 9, in this figure the following column images are depicted:

a)Dorsal hand vein thermal image.

b)ROI extraction, noise reduction and normalization where each hand image is enhanced in order to obtain a better contrast.

c)Hand vein segmentation using local thresholding where each segmented vein region is in black color.

d)Thinning of the vein regions obtained after segmentation.

e)Bifurcation and end points found as minutiae. For a better appreciation of the points, these results are show in negative with respect to the thinning image result.

Based on the results shown in Fig. 9, it can be seen that in most of the cases, the main structures of the hand vein are found and also their respective bifurcation and end points. In addition, the proposed method can be used for images where there exists low contrast in the hand vein image such as the image shown in the bottom of Fig. 9. 

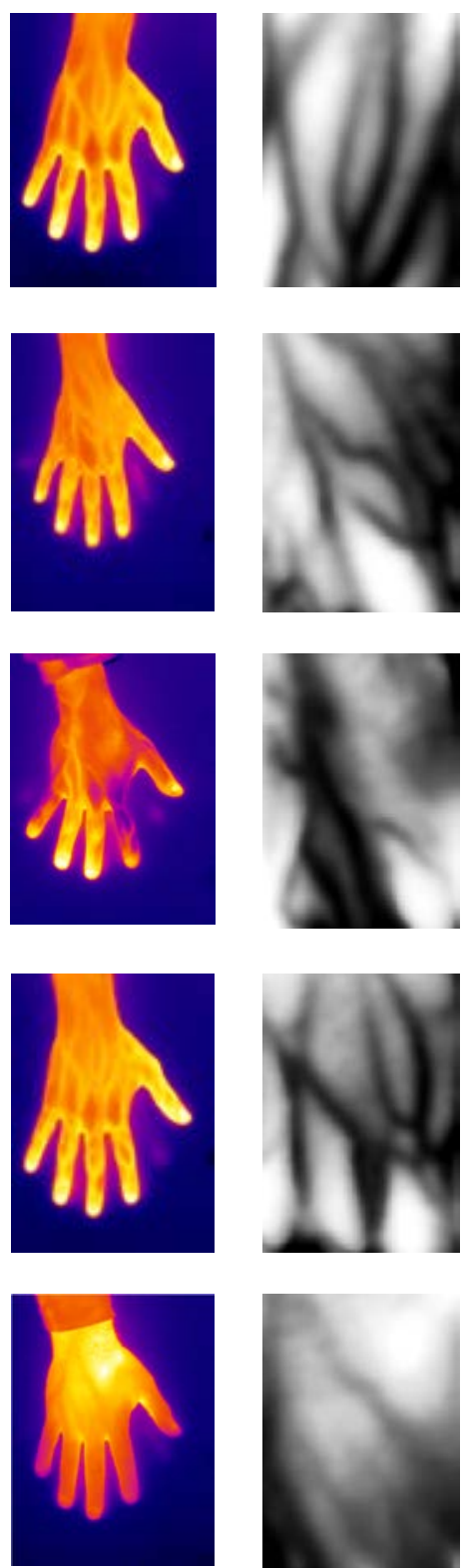

a)
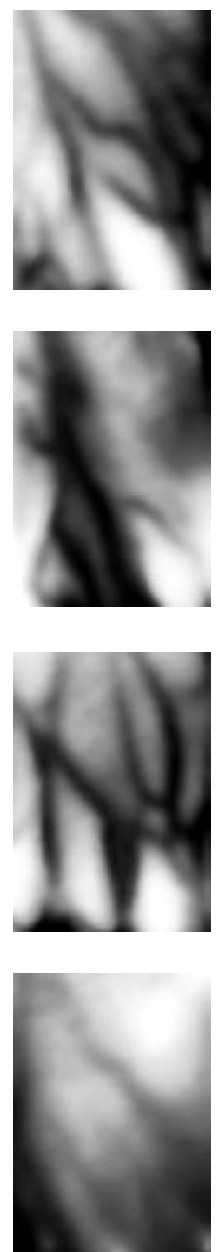
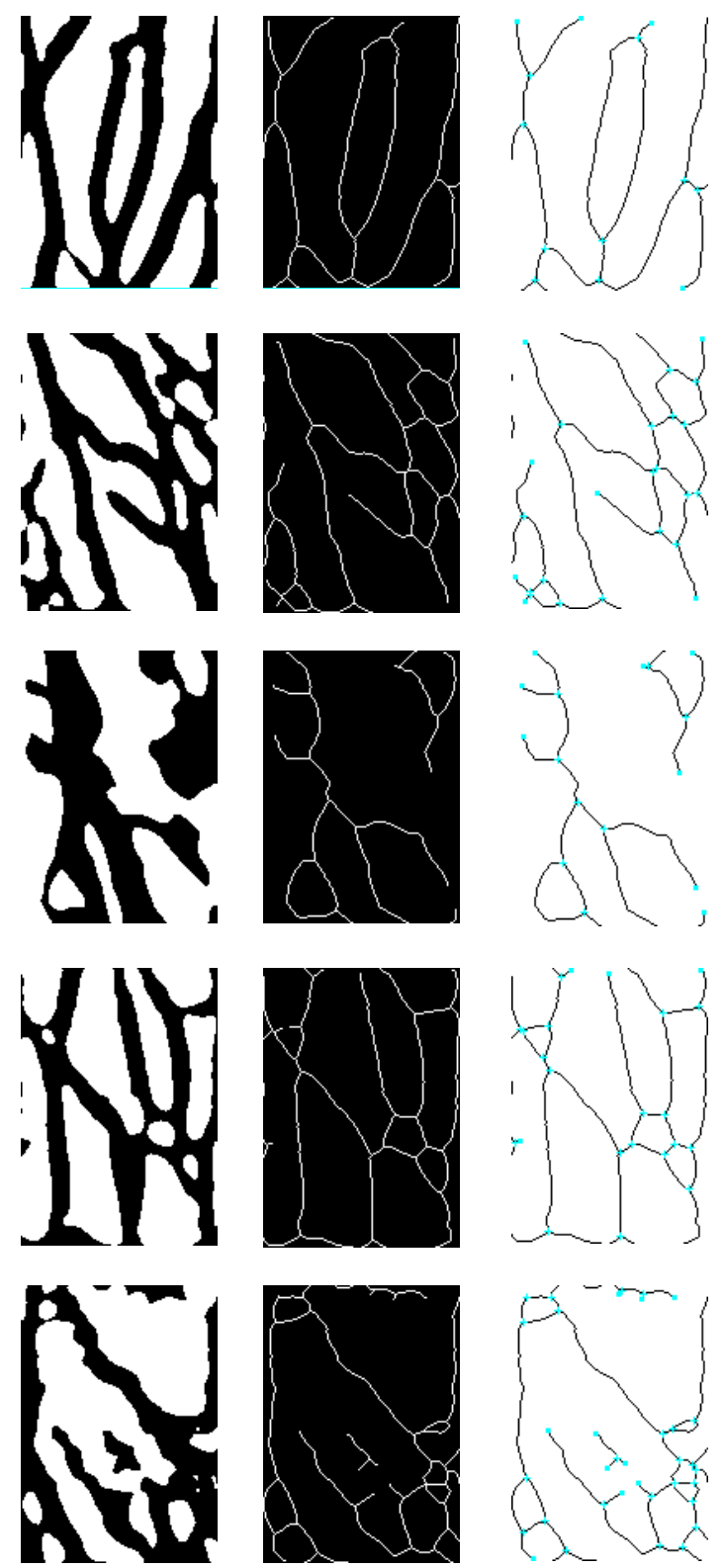

d)

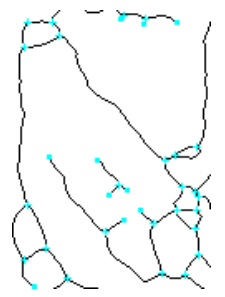

c)

e)

Fig. 9. a)Thermal hand dorsal images. b) ROI enhanced and normalized. c)Segmentation obtained over images in b). c)Hand vein thinning. d)Bifurcation and end points found 


\section{Conclusions}

This paper proposes a technique to segment the vein patterns in the human hand, and then extracting minutiae points. This technique may be used to process low contrast images, even when it is not a global solution because it can be affected by motion or rotation; it is enough to be used in several cases. It is very important to highlight that with this approach is possible to extract features (minutiae) from hand vein thermal images with different level of low contrast. Hand vein is considered as biometric because it has a special characteristic since for recognizing a person through this biometric it is necessary that the person stays alive.

This initial research work is related to the pre-processing, ROI extraction and feature extraction stages in a biometric system. Then, as future work we will analyze our approach applying/proposing other thresholding and feature extraction methods in order to construct training sets to be used by classifiers such as Hamming k-Nearest Neighbors, Neural Networks and Support Vector Machines in the recognition step. Finally, an analysis of the recognition results is also included in our future work.

Acknowledgment. This work was supported by the projects OLLJ-ING14-IVIEPBUAP (Puebla, Mexico) and PROMEP/103.5/12/7954UPSIN-PTC-015 (Sinaloa, Mexico).

\section{References}

1. Jain, A. K., Ross, A. A., Nandakumar, K.: Introduction to Biometrics. Springer, (2012)

2. Samaria, F., Harter, A.: Parameterization of a Stochastic Model for Human Face Identification. In: Proceedings of 2nd IEEE Workshop on Applications of Computer Vision, 138142 (1994)

3. CASIA iris database.: Institute of Automation, Chinese Academy of Sciences. http://biometrics.idealtest.org

4. Fierrez, J., Galbally, J., Ortega-Garcia, J., Freire, M. R., Alonso-Fernández, F., Ramos, D., Gracia-Roche, J.: BiosecurID: a multimodal biometric database. Pattern Analysis and Applications, 13(2), 235-246(2010)

5. Wang, L., Leedham, G.: Near and far infrared imaging for vein pattern biometrics. In: Proceedings of the IEEE International Conference on Video and Signal Based Surveillance, p. 52. IEEE Computer Society (2006)

6. Jain, A. K., Bolle, R. M., Pankatni, S.: Biometrics: Personal Identification in Networked Society, Kluwer Academic Publishers, Dordrecht (1999)

7. MacGregor, P., Welford, R.: Veincheck: imaging for security and personnel identification, Adv. Imaging 6(7), 52-56 (1991)

8. Hawkes, P. L., Clayden, D. O.: Veincheck research for automatic identification of people, in: Hand and Fingerprint Seminar at NPL, 230-233 (1993)

9. Wang, L., Leedham, G., Siu-Yeung, C. D.: Minutiae feature analysis for infrared hand vein pattern biometrics. Pattern recognition, 41(3), 920-929 (2008)

10. Romen, S. T., Roy, S., Imocha, S. O., Sinam, T., Singh, K. M.: A New Local Adaptive Thresholding Technique in Binarization. International Journal of Computer Science Issues 8(6) No. 2, 271-277 (2011) 
Ignacio Irving Morales-Montiel, J. Arturo Olvera-López, Manuel Martín-Ortíz, et al.

11. Zhang, T. Y., Suen, C. Y.: A fast parallel algorithm for thinning digital patterns. Communications of the ACM, 27(3), 236-239 (1984)

12. Mishra, K. N., Agrawal, P. C., Tripathi, A., Garg, V.: Minutiae Distances and Orientation Fields Based Thumbprint Identification of Identical Twins. International Journal of Image, Graphics and Signal Processing, 5(2), 51-59 (2013)

13. Faundez-Zanuy, M., Mekyska, J., Font-Aragonés, X.: A new hand image database simultaneously acquired in visible, near-infrared and thermal spectrums. Cognitive Computation, 6(2), 230-240 (2014) 